\title{
A reciprocating motion-driven rotation mechanism for the ATP synthase
}

\author{
Jiafeng Liu ${ }^{1}$, Xinmiao $\mathrm{Fu}^{1,2^{*}} \&$ Zengyi Chang ${ }^{1,2^{*}}$ \\ ${ }^{1}$ State Key Laboratory of Protein and Plant Gene Research, School of Life Sciences, Peking University, Beijing 100871, China; \\ ${ }^{2}$ Center for Protein Science, Peking University, Beijing 100871, China
}

Received October 8, 2015; accepted November 27, 2015; published online December 29, 2015

\begin{abstract}
The ATP synthase (having a typical subunit composition of $\alpha_{3} \beta_{3} \gamma \delta \varepsilon a b_{2} c_{8-15}$ ) employs an intriguing rotary mechanism for the generation of ATP from ADP and $\mathrm{P}_{\mathrm{i}}$, using energy stored in a transmembrane proton gradient. The conventional rotary model, although being generally accepted, remains difficult to explain certain experimental observations. Here we propose an alternative rotary model for the ATP synthase such that what rotates is the catalytic $\alpha_{3} \beta_{3}$ cylinder rather than the central stalk and the membrane-embedded c-ring. Specifically, the membrane translocation of protons would induce a cycled conformational change in the c-ring, leading to a reciprocating motion of the attached central stalk, which in turn drives the unidirectional rotation of the $\alpha_{3} \beta_{3}$ cylinder. Such a reciprocating motion-driven rotation mechanism is somehow analogous to the working mechanism of a retractable click ballpoint pen. Our new model not only explains the experimental observations that have been difficult to reconcile with the conventional model but also avoids its theoretical illogicality.
\end{abstract}

ATP synthase, rotational catalysis, rotary model

Citation: Liu, J., Fu, X., and Chang Z. (2016). A reciprocating motion-driven rotation mechanism for the ATP synthase. Sci China Life Sci 59, 44-48. doi: $10.1007 / \mathrm{s} 11427-015-4995-0$

\section{INTRODUCTION}

The ATP synthase (also known as the $\mathrm{F}_{1} \mathrm{~F}_{0}$-ATPase), being recognized as the principle energy transformer in living organisms, is a membrane-integrated multi-subunit protein complex (von Ballmoos et al., 2009). It catalyzes the synthesis of ATP from ADP and $\mathrm{P}_{\mathrm{i}}$ by utilizing the electrochemical potential energy stored in a transmembrane proton gradient (Mitchell, 1961). The overall architecture of ATP synthase, being highly conserved from bacteria to human, is composed of the $F_{o}$ and $F_{1}$ parts (von Ballmoos et al., 2008). The membrane-integrated $F_{o}$ part (having a typical subunit composition of $\mathrm{ab}_{2} \mathrm{c}_{8-15}$ ) contains the proton channel, while the membrane-extrinsic $F_{1}$ part (having a typical subunit composition of $\alpha_{3} \beta_{3} \gamma \delta \varepsilon$ ) contains the catalytic sites for ATP

*Corresponding author (email: fuxinmiao@pku.edu.cn; changzy@pku.edu.cn) synthesis (Abrahams et al., 1994; Stock et al., 1999).

It has been generally accepted that the catalysis of the ATP synthase occurs via an intriguing rotary mechanism (Junge and Nelson, 2015). According to the current view of this mechanism, the membrane-embedded c-ring is driven to rotate unidirectionally by the downhill proton translocation along a channel formed between the a-subunit and the c-ring (Duncan et al., 1995; Junge et al., 1996; Vik and Antonio, 1994), and the c-ring in turn drives the rotation of the attached central stalk (composing of the $\gamma$ - and $\varepsilon$ subunits), which sequentially actuates the conformational change of the three catalytic $\alpha \beta$ units for synthesizing ATP (Boyer, 1997).

In retrospect, such a rotary model has been evolved over years of conceptual contemplations. Initially in early 1980s, Paul Boyer's group proposed that "A relative rotational movement of $\beta$-subunits and a control subunit core could merit consideration" to explain how the three catalytic 
$\beta$-subunits that interact with one single $\gamma$-subunit could participate in catalysis identically (Gresser et al., 1982). Later in 1994, Vik and Antonio proposed that the oligomer of the c-subunits rotates with respect to the a-subunit to explain how the protons are translocated across the ATP synthase (Vik and Antonio, 1994). Subsequently, relative rotation between the $\gamma$-subunit of central stalk and the $\alpha_{3} \beta_{3}$ hexamer was demonstrated by intersubunit chemical crosslinking (Duncan et al., 1995), fluorescence anisotropy measurements (Sabbert et al., 1996) or epifluorescent microscopy (Noji et al., 1997). These produced the current picture of the rotary model of the ATP synthase as usually documented in textbooks.

\section{RESULTS}

\section{Defects of the conventional model}

Notwithstanding the general acceptance of the conventional rotary model of the ATP synthase, there remain aspects that are difficult to reconcile with (Walker, 2013). For example, it is difficult to visualize how one round rotation of the central stalk that was reported to proceed via three $120^{\circ}$-steps (Yasuda et al., 2001) is synchronized with one round rotation of the c-ring that proceeds via a number of steps that equal to the number of subunits in the c-ring (Düser et al., 2009). In particular, when the number of subunits of the c-ring of the ATP synthase is non-integer multiple of 3 (e.g. $8,10,11,13$ or 14 ), as observed in certain organisms (Meier et al., 2005; Stock et al., 1999; Vollmar et al., 2009; Watt et al., 2010), one $120^{\circ}$-step of the central stalk rotation would have to correlate to a non-integer number of steps of the c-ring rotation, a scenario that is too complicated to contemplate and apparently disobey the rule of parsimony.

In addition, there should be theoretically at least three different orientations for the central stalk in reference to the stationary peripheral stalk (composing the a- and the bsubunits) in the resolved structure of ATP synthase, according to the conventional rotary model. Nevertheless, the central stalk was found to adopt only one specific orientation in the determined X-ray crystal structure of a sub-complex of the bovine ATP synthase (Rees et al., 2009) (Figure 1A). Similarly, the central stalk of the ATP synthase from an archaea (Lau and Rubinstein, 2011) (Figure 1B) and mitochondria of a green alga (Allegretti et al., 2015) was both found to adopt a single orientation as revealed by cryo-electron microscopy analysis. Remarkably, for the structures of all these ATP synthases, we found that their central stalks apparently adopted the same orientation in reference to their peripheral stalks (Figure 1). These experimental observations raise a possibility that the central stalk actually does not rotate in reference to the peripheral stalk.

\section{The lack of direct evidence showing that the c-ring/central stalk rotates}

Though the relative rotation between the c-ring/central stalk
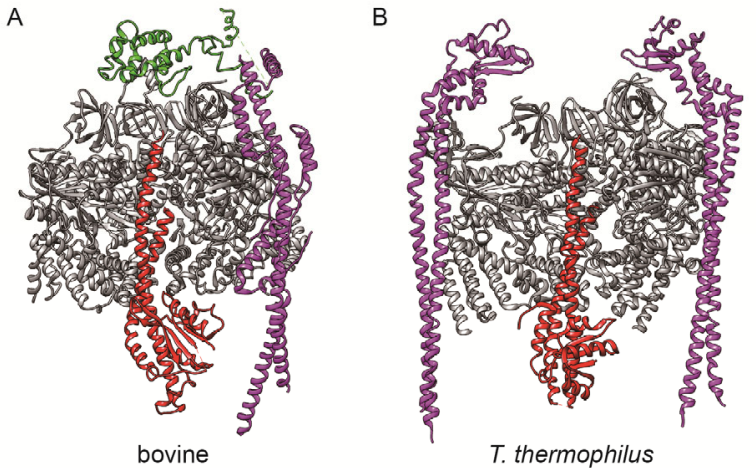

Figure 1 The central stalk adopts a single orientation in reference to the membrane-integrated peripheral stalk in the ATP synthase. A, The X-ray crystal structure of the $\mathrm{F}_{1}$-peripheral stalk complex from bovine mitochondrial ATP synthase (Dickson et al., 2006; Rees et al., 2009) (PDB ID: 2 WSS and 2CLY). B, The atomic models of the $\mathrm{F}_{1}$ and the peripheral stalk subunits as fitted into the cryo-electron microscopy map of the ATP synthase from Thermus thermophiles (Lau and Rubinstein, 2011) (PDB ID: 3J0J). The structures are shown in ribbon representation. The central stalks (containing the $\gamma$-subunit in the bovine or the D- and F-subunits in the $T$. thermophiles ATP synthases) are colored red and the peripheral stalks (containing the b-, d- and $\mathrm{F}_{6}$-subunits in the bovine, or the $\mathrm{E}$ - and G-subunits in the T. thermophiles ATP synthases) are colored purple. The $\delta$-subunit in panel A) is colored green. The two sets of the $\alpha \beta$-subunits in both panels are all colored gray, with one set of the $\alpha \beta$-subunits facing the reader being not displayed in both panels.

and the $\alpha_{3} \beta_{3}$ hexamer was clearly observed by multiple in vitro studies (Duncan et al., 1995; Noji et al., 1997; Sabbert et al., 1996), there lacks direct experimental evidence showing that the c-ring/central stalk actually rotates in reference to the membrane-integrated peripheral stalk for the ATP synthase. For example, Börsch et al. concluded that the $\gamma$-subunit of the central stalk rotates relative to the b-subunit based on the observation of three states with different intramolecular single molecule fluorescence resonance energy transfer (FRET) efficiencies between these two subunits (Börsch et al., 2002). Martin et al. concluded that the c-ring rotates based on the observation of a cycled orientation change of a nanorod being attached to the c-ring of an ATP synthase molecule embedded in lipid bilayer nanodiscs (Martin et al., 2015). These experimental data certainly reflected a cycled conformational change of the c-ring/central stalk, but whether the c-ring/central stalk rotates, as explained by these authors, is uncertain because the rotation motion could only be considered as a special case of such cycled conformational change. In a recent electron cryomicroscopy analysis, the authors claimed that they obtained three rotational states of the yeast V-ATPase, but they were biased in this study by preassuming the existence of such three states and thus artificially classified the particle images into three groups before performing their computational reconstruction (Zhao et al., 2015).

\section{A reciprocating motion-driven rotation mechanism}

Here we propose an alternative rotary model in which what 
rotates is the cylinder of the $\alpha_{3} \beta_{3}$ hexamer rather than the c-ring and the central stalk. In proposing this reciprocating motion-driven rotation mechanism, as illustrated schematically in Figure 2A, we are inspired by how a retractable click ballpoint pen works, in which the reciprocating motion of a push rod is transformed to the rotational motion of a cam through an inclined interface. Specifically, for the ATP synthase, translocation (i.e. the binding and releasing) of protons through the channel between the c-ring and the a-subunit induces a cycled conformational change in the c-ring, which leads to the back and forth motion of the attached central stalk in a direction perpendicular to the membrane, in turn driving a unidirectional rotation of the $\alpha_{3} \beta_{3}$ hexamer.

According to our model, the direct motion transmission from the c-ring to the central stalk would require the $\gamma$-subunit of the central stalk to contact the c-ring at a site close to the proton channel. Our analyses of the previously reported structures of ATP synthase do reveal, as illustrated in Figure 3A, that the $\gamma$-subunit contacts the c-ring by interacting with the few c-subunits near the a-subunits, where the proton translocation channel apparently sites.

More importantly, for the back-and-forth motion of the central stalk (as the push rod) to drive the rotation of the $\alpha_{3} \beta_{3}$ hexamer (as the cam), it would rely on a critical inclined interface between them (refer to Figure 2A). Remarkably, a contact between the $\gamma$-subunit and the $\beta$-subunit, as indicated in Figure $3 \mathrm{~B}$, apparently serves as such a putative inclined interface. Although being ignored before, the functional importance of this interface is strongly implicated by the fact that it involves the most conserved Asp/Asn-Arg/ Lys-Gly-Leu-Cys-Gly sequence along the whole polypeptide chain of the $\gamma$-subunit (Figure 3C).

Another critical component for our model to work is the presence of a cap that is able to prevent the $\alpha_{3} \beta_{3}$ hexamer to shift position during its rotation upon pushing by the central stalk (refer to Figure 2A). In this regard, the $\delta$-subunit (being called the OSCP-subunit in the mitochondrial ATP synthase; refer to Figure 1A) would ideally act as such a cap. Meanwhile, the $\delta$-subunit would also serve as a hinge joining the rotating $\alpha_{3} \beta_{3}$ hexamer to the stationary peripheral stalk.

Apparently, our model is highly compatible with the structure information of the ATP synthase as provided by electron cryomicroscopy and X-ray crystallography. It follows that we are able to generate a structure-based synthetic animation to display the reciprocating motion-driven rotation mechanism of the ATP synthase, as shown in the supplementary movie (one picture of which is shown in Figure 2B).

\section{DISCUSSION}

Here we propose a reciprocating motion-driven rotation mechanism for the ATP synthase, in which what rotates is the cylinder of the $\alpha_{3} \beta_{3}$ hexamer instead of the c-ring and the central stalk. Our model not only explains the experimental observations that have been difficult to reconcile with the conventional rotary model but also avoids its theoretical illogicality. For example, the synchronization between the rotations of the c-ring and the central stalk in the context of the conventional model is no longer an issue in our model. Additionally, the number of protons translocated for generating each round of $360^{\circ}$ rotation (i.e., for making three ATP molecules) does not have to be equal to the exact number of subunits in the c-ring. Instead, such a c-ring oligomer, made of various numbers of c-subunits in different species, might be viewed as a symmetric entity that is able to maintain stability during its cycled conformational change in participating proton translocation.
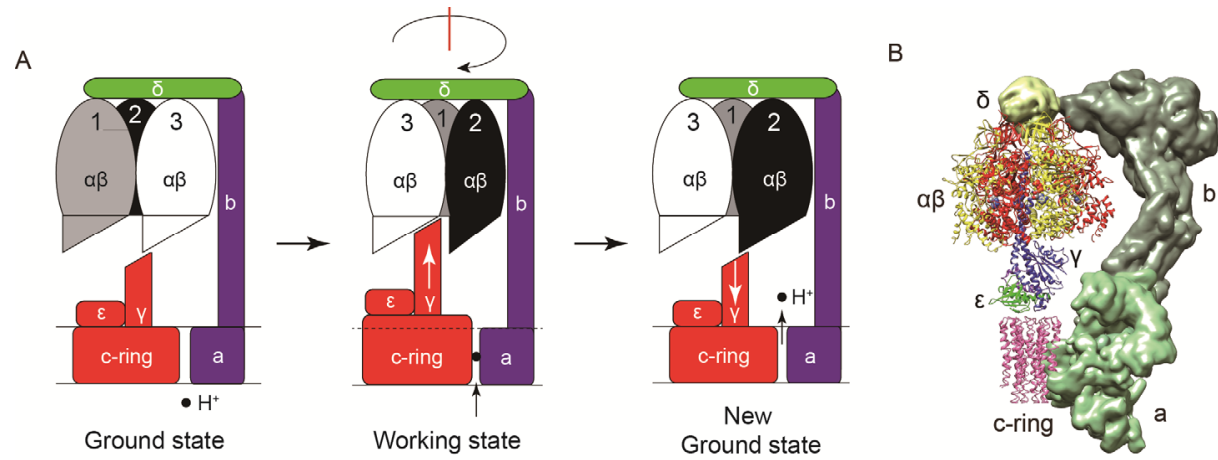

Figure 2 A schematic illustration of the reciprocating motion-driven rotation mechanism for the ATP synthase to reflect how the back and forth linear motion of the central stalk is transformed to the circular rotational motion of the $\alpha_{3} \beta_{3}$ hexamer (A), and a static state of a structure-based animation of this mechanism (B). A, In the "working state", the binding of proton(s) to the channel triggers a conformational change of the c-ring, which results in an upward motion of the central stalk that in turn, through the inclined interface, drives the rotation of the $\alpha_{3} \beta_{3}$ hexamer, whose position shift is prevented by the $\delta$-subunit cap. In the "new ground state", the releasing of proton(s) from the channel resets the c-ring and the attached central stalk, returning the whole enzyme to its "ground state" and getting the ATP synthase ready for another cycle of rotational catalysis. B, The animation was generated based on the electron cryomicroscopy map of the Polytomella F-type ATP synthase (Allegretti et al., 2015) and the crystal structure of bovine ATP synthase (Rees et al., 2009). 

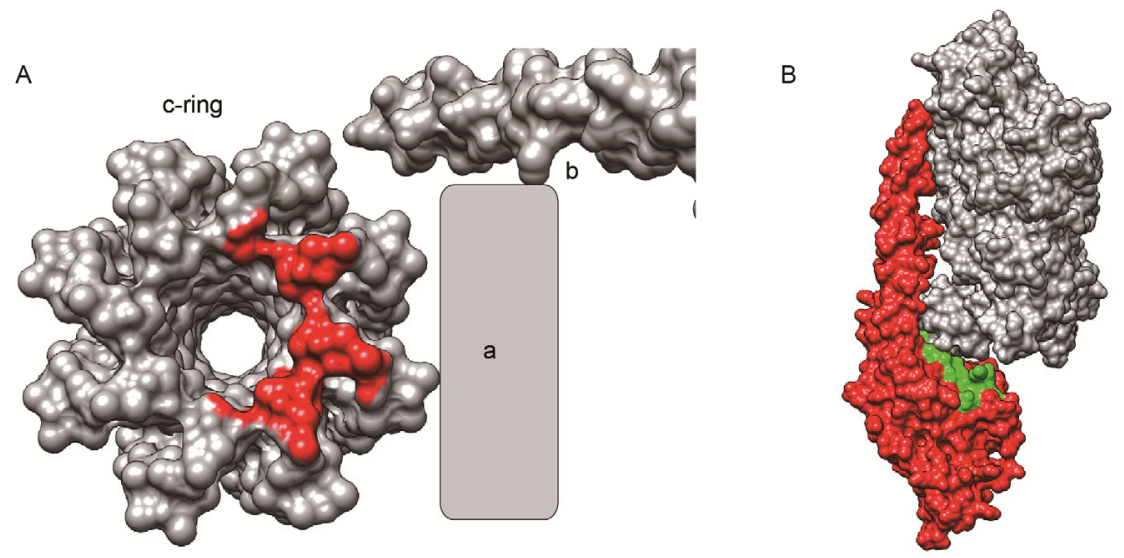

C

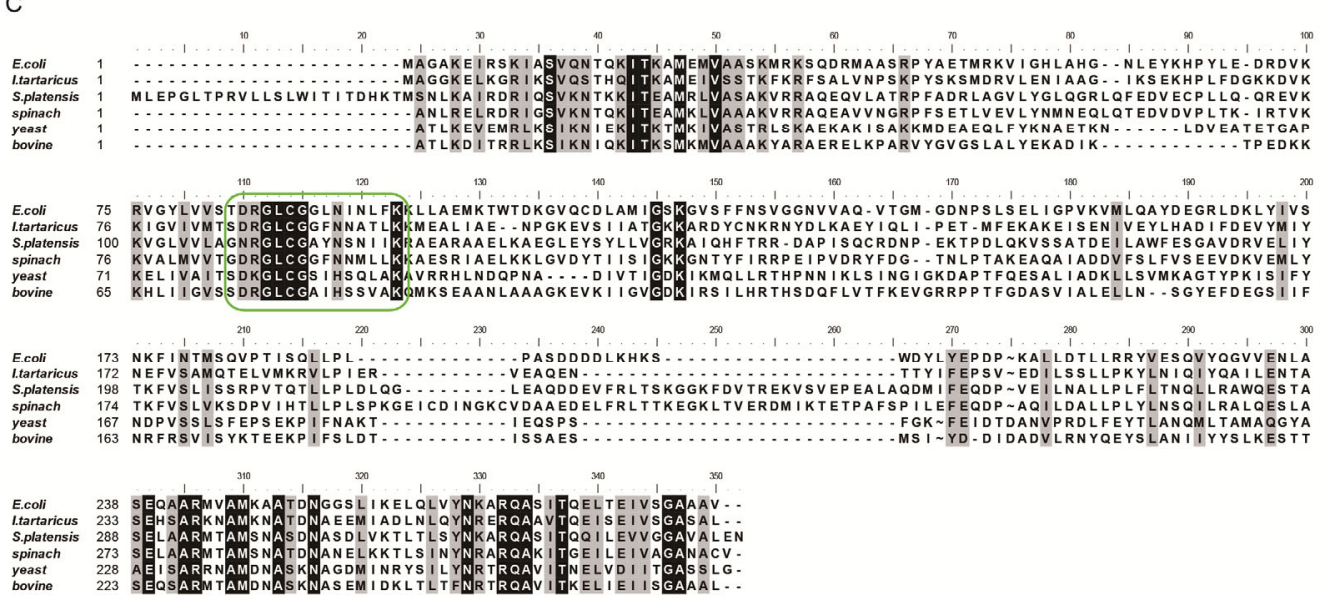

Figure 3 The $\gamma$-subunit of the central stalk interacts with the c-ring in an asymmetric manner (A) and with the $\alpha \beta$-dimer through an inclined surface (B), composing of the most conserved stretch of amino acid residues (C). A, Displayed is the c-ring surface (colored red) that interacts with the $\gamma$-subunit, as identified in the bovine ATP synthase (Watt et al., 2010) (PDB ID: 2XND). The structures are displayed in solid representation, with the c-ring, the a- and b-subunits all being colored gray. Location of the b-subunit is deduced from the determined crystal structure of bovine ATP synthase (Rees et al., 2009) (PDB ID:2WSS), and that of the a-subunit is deduced from the cryo-electron microscopy of mitochondrial ATP synthase (Allegretti et al., 2015). B, A $\gamma$-subunit interacting with a $\alpha \beta$-dimer, as taken from the determined crystal structure of bovine ATP synthase (Rees et al., 2009) (PDB ID: 2WSS). The $\alpha \beta$-dimer is colored gray and the $\gamma$-subunit red, with the interaction surface of the $\gamma$-subunit colored green. C, Sequence alignment of the $\gamma$-subunits of the ATP synthase from the indicated organisms. The residues composing the interaction surface with the $\alpha \beta$-dimer (colored green in (B)) is circled by the green frame.

An additional major issue that we believe to exist in the conventional model is the potential disturbance on the membrane structure resulting from the high-speed rotation (up to 100 rounds per second) of the membrane-embedded c-ring. Such disturbance would be ignorable given that the c-ring is proposed not to rotate in our model.

What distinguishes our reciprocating motion-driven rotation mechanism from the conventional model is whether the $\alpha_{3} \beta_{3}$ hexamer or the c-ring rotates in reference to the membrane-integrated peripheral stalk. As a matter of fact, under in vitro conditions, the rotation of the $\alpha_{3} \beta_{3}$ hexamer, as driven either by hydrolysis of ATP (Nishio et al., 2002; Tanabe et al., 2001) or by a transmembrane proton gradient (Watanabe et al., 2013), was effectively detected in the holo-ATP synthase when the c-ring was immobilized. According to our model, these data solely indicate the rotation of the $\alpha_{3} \beta_{3}$ hexamer, without requiring the membraneintegrated peripheral stalk to rotate along as having to be explained according to the conventional model. Nevertheless, it would be technically difficult to determine whether it is the $\alpha_{3} \beta_{3}$ hexamer or the c-ring that actually rotates in living cells and it awaits new approaches to resolve this critical issue. One possible solution to this issue is that, in the aformentioned experimental system (Watanabe et al., 2013), attach the probe to the b-subunit of the peripheral stalk, instead of on the $\alpha_{3} \beta_{3}$ hexamer, and examine whether the probe rotates (it would rotate according to the conventional model, but not according to our model).

Compliance and ethics The author(s) declare that they have no conflict of interest.

Acknowledgements We thank Mr. Yang Liu, Ms. Jiayu Yu, Mr. Haotian Xiao, Mr. Rui Wang and Ms. Feng Jin of Peking University for valuable discussions. Molecular graphics and analyses were performed with the University of California, San Francisco Chimera package. This work was 
supported by research grants from the National Basic Research Program of China (2012CB917300 to Zengyi Chang and Xinmiao Fu), and the National Natural Science Foundation of China (31470766 and 31170738 to ZYC, 31270804 and 31570778 to Xinmiao Fu).

Author contributions Jiafeng Liu, Xinmiao Fu and Zengyi Chang prepared the manuscript.

Abrahams, J.P., Leslie, A.G.W., Lutter, R., and Walker, J.E. (1994). Structure at $2.8 \mathrm{~A}$ resolution of $\mathrm{F}_{1}$-ATPase from bovine heart mitochondria. Nature 370, 621-628.

Allegretti, M., Klusch, N., Mills, D.J., Vonck, J., Kuhlbrandt, W., and Davies, K.M. (2015). Horizontal membrane-intrinsic $\alpha$-helices in the stator a-subunit of an F-type ATP synthase. Nature 521, 237-240.

von Ballmoos, C., Cook, G.M., and Dimroth, P. (2008). Unique rotary ATP synthase and its biological diversity. Annu Rev Biophys 37, 43-64.

von Ballmoos, C., Wiedenmann, A., and Dimroth, P. (2009). Essentials for ATP synthesis by $\mathrm{F}_{1} \mathrm{~F}_{0}$ ATP synthases. Annu Rev Biochem 78, 649-672.

Börsch, M., Diez, M., Zimmermann, B., Reuter, R., and Gräber, P. (2002). Stepwise rotation of the $\gamma$-subunit of $\mathrm{EF}_{0} \mathrm{~F}_{1}$-ATP synthase observed by intramolecular single-molecule fluorescence resonance energy transfer 1. FEBS Lett 527, 147-152.

Boyer, P.D. (1997). The ATP synthase-a splendid molecular machine. Annu Rev Biochem 66, 717-749.

Dickson, V.K., Silvester, J.A., Fearnley, I.M., Leslie, A.G.W., and Walker, J.E. (2006). On the structure of the stator of the mitochondrial ATP synthase. EMBO J 25, 2911-2918.

Duncan, T.M., Bulygin, V. V, Zhou, Y., Hutcheon, M.L., and Cross, R.L. (1995). Rotation of subunits during catalysis by Escherichia coli $\mathrm{F}_{1}$-ATPase. Proc Natl Acad Sci USA 92, 10964-10968.

Düser, M.G., Zarrabi, N., Cipriano, D.J., Ernst, S., Glick, G.D., Dunn, S.D., and Börsch, M. (2009). 36 degrees step size of proton-driven c-ring rotation in $\mathrm{F}_{0} \mathrm{~F}_{1}-\mathrm{ATP}$ synthase. EMBO J 28, 2689-2696.

Gresser, M.J., Myers, J.A., and Boyer, P.D. (1982). Catalytic site cooperativity of beef heart mitochondrial $F_{1}$ adenosine triphosphatase. Correlations of initial velocity, bound intermediate, and oxygen exchange measurements with an alternating three-site model. J Biol Chem 257, 12030-12038.

Junge, W., and Nelson, N. (2015). ATP Synthase. Annu Rev Biochem 84, 631-657.

Junge, W., Sabber, D., and Engelbrecht, S. (1996). ATP-synthesis. Rotatory catalysis by F-ATPase: real-time recording of intersubunit rotation. Berichte Der Bunsengesellschaft Für Phys Chemie 100, 2014-2019.

Lau, W.C.Y., and Rubinstein, J.L. (2011). Subnanometre-resolution structure of the intact Thermus thermophilus $\mathrm{H}^{+}$-driven ATP synthase. Nature 481, 214-218.

Martin, J., Hudson, J., Hornung, T., and Frasch, W.D. (2015). F $F_{0}$-driven rotation in the ATP synthase direction against the force of $F_{1}$ ATPase in the $\mathrm{F}_{\mathrm{o}} \mathrm{F}_{1}$ ATP synthase. J Biol Chem 290, 10717-10728.

Meier, T., Polzer, P., Diederichs, K., Welte, W., and Dimroth, P. (2005). Structure of the rotor ring of F-type $\mathrm{Na}^{+}$-ATPase from Ilyobacter tartaricus. Science 308, 659-662.

Mitchell, P. (1961). Coupling of phosphorylation to electron and hydrogen transfer by a chemi-osmotic type of mechanism. Nature 191, 144-148.

Nishio, K., Iwamoto-Kihara, A., Yamamoto, A., Wada, Y., and Futai, M. (2002). Subunit rotation of ATP synthase embedded in membranes: a or beta subunit rotation relative to the $\mathrm{c}$ subunit ring. Proc Natl Acad Sci USA 99, 13448-13452.

Noji, H., Yasuda, R., Yoshida, M., and Kinosita, K. (1997). Direct observation of the rotation of $F_{1}$-ATPase. Nature 386, 299-302.

Rees, D.M., Leslie, A.G.W., and Walker, J.E. (2009). The structure of the membrane extrinsic region of bovine ATP synthase. Proc Natl Acad Sci USA 106, 21597-21601.

Sabbert, D., Engelbrecht, S., and Junge, W. (1996). Intersubunit rotation in active F-ATPase. Nature 381, 623-625.

Stock, D., Leslie, A.G.W., and Walker, J.E. (1999). Molecular architecture of the rotary motor in ATP synthase. Science 286, 1700-1705.

Tanabe, M., Nishio, K., Iko, Y., Sambongi, Y., Iwamoto-Kihara, A., Wada, Y., and Futai, M. (2001). Rotation of a complex of the $\gamma$ subunit and c ring of Escherichia coli ATP synthase. The rotor and stator are interchangeable. J Biol Chem 276, 15269-15274.

Vik, S.B., and Antonio, B.J. (1994). A mechanism of proton translocation by $F_{1} F_{0}$ ATP synthases suggested by double mutants of the a subunit. $J$ Biol Chem 269, 30364-30369.

Vollmar, M., Schlieper, D., Winn, M., Büchner, C., and Groth, G. (2009). Structure of the $c_{14}$ rotor ring of the proton translocating chloroplast ATP synthase. J Biol Chem 284, 18228-18235.

Walker, J.E. (2013). The ATP synthase: the understood, the uncertain and the unknown. Biochim Soc Trans 41, 1-16.

Watanabe, R., Tabata, K. V, Iino, R., Ueno, H., Iwamoto, M., Oiki, S., and Noji, H. (2013). Biased Brownian stepping rotation of $F_{0} F_{1}$-ATP synthase driven by proton motive force. Nat Commun 4, 1631.

Watt, I.N., Montgomery, M.G., Runswick, M.J., Leslie, A.G.W., and Walker, J.E. (2010). Bioenergetic cost of making an adenosine triphosphate molecule in animal mitochondria. Proc Natl Acad Sci USA 107, 16823-16827.

Yasuda, R., Noji, H., Yoshida, M., Kinosita, K., and Itoh, H. (2001). Resolution of distinct rotational substeps by submillisecond kinetic analysis of $F_{1}$-ATPase. Nature 410, 898-904.

Zhao, J., Benlekbir, S., and Rubinstein, J.L. (2015). Electron cryomicroscopy observation of rotational states in a eukaryotic V-ATPase. Nature 521, 241-245.

Open Access This article is distributed under the terms of the Creative Commons Attribution License which permits any use, distribution, and reproduction in any medium, provided the original author(s) and source are credited.

\section{SUPPORTING INFORMATION}

\section{Supplementary movie}

The supporting information is available online at life.scichina.com and link.springer.com. The supporting materials are published as submitted, without typesetting or editing. The responsibility for scientific accuracy and content remains entirely with the authors. 